\title{
Comparison of the diagnostic value of cervical cytology and HPV HR DNA testing for the diagnosis of low-grade and high-grade squamous intraepithelial lesions across different age groups
}

\author{
Aleksandra Paluszkiewicz ${ }^{1}$, Dominik Pruski ${ }^{1}$, ${ }^{\text {, Kinga Iwaniec }}{ }^{1}$, Witold Kędziaa ${ }^{1,2}$ \\ ${ }^{1}$ Division of Gynecology, Department of Perinatology and Gynecology, Gynecology and Obstetrics Clinical Hospital, \\ Karol Marcinkowski University of Medical Sciences, Poznan, Poland \\ ${ }^{2}$ Laboratory of Cervical Pathophysiology, Gynecology and Obstetrics Clinical Hospital, \\ Karol Marcinkowski University of Medical Sciences, Poznan, Poland
}

\begin{abstract}
Objectives: To assess the diagnostic value of cervical cytology and HPV HR DNA testing for the diagnosis of low grade and high-grade squamous intraepithelial lesions across different age groups.

Material and methods: The study included 1103 patients, age 25-70 years. All patients underwent in-depth diagnostic tests following either an abnormal Pap test result or a clinically suspicious cervical lesion. In all women the following examinations were performed: a molecular test detecting 14 high-risk types of HPV, a colposcopy examination, as well as directed-biopsy of the cervix. The studied population was subdivided into four age groups.

Results: It was observed that the percentage of high grade squamous intraepithelial lesions (HSIL) and cancers increased with women's age. Sensitivity of both methods for detecting high-grade squamous intraepithelial lesions was highest for women aged 40-49 years. Sensitivity values of HPV testing was higher than that of cervical cytology among women under age 50 .

Conclusions: Specificity of HPV testing increased significantly with age of women and was several fold higher across all age groups than the specificity of cervical cytology.
\end{abstract}

Key words: Pap test, HPV testing, low-grade squamous intraepithelial lesions (LSIL), high-grade squamous intraepithelial lesions (HSIL), age groups

\section{INTRODUCTION}

Retrospective analysis of the incidence rate of cervical cancer in Poland shows, that the epidemiological situation has improved over the last thirty years, especially during the last decade. Nevertheless, it is estimated that over 3000 new cervical cancers are being diagnosed each year and over half of the women die from the disease [1]. Cervical intraepithelial neoplasia can effectively undergo conservative treatment, which is of a special value for women of reproductive age.

Since mid-nineties the incidence and mortality rates of cervical cancer have decreased in Poland [2]. After 2005, the introduction of the Screening Program for the Prophylaxis and Early Diagnosis of Cervical Cancer laid the groundwork for dynamic changes. Cytology-based screening was indicated for women aged 25-59 years.

The primary purpose of cervical screening programs is to diagnose precancerous lesions of the cervix which, according to FIGO of 1988, were classified as either low- (CIN 1), mid- (CIN 2) or high-grade cervical intraepithelial neoplasia (CIN 3). Cervical intraepithelial neoplasia are defined by both morphological criteria and architectural features of epithelial layers. Until recently, these lesions - CIN 2 and CIN 3, 
were defined as true precancerous lesions of the cervix. In 2012, the College of American Pathologists together with the American Society of Colposcopy and Cervical Pathology initiated a project, which introduced new nomenclature of HPV-related squamous lesions of anogenital region (LAST - The Lower Anogenital Squamous Terminology Project). Since the diagnosis of CIN 2 is characterized by low repetition and a high percentage of these lesions are reclassification as either CIN 1 or CIN 3, the LAST Project supports a two-tiered nomenclature of squamous intraepithelial lesions, distinguishing low-grade squamous intraepithelial lesions (LSIL) and high-grade squamous intraepithelial lesions (HSIL). Lesions previously classified as CIN 2 should be tested immunohistochemically in order to detect the expression of $\mathrm{p} 16$ protein. Lesions negative for $\mathrm{p} 16$ should be classified as LSIL, while lesions positive for $\mathrm{p} 16$ should be classified as HSIL $[3,4]$.

For many years, there has been an ongoing debate on the improvement of diagnostic tools to detect precancerous states of the cervix. Future research should focus on the age at which screening should be initiated, as well as the optimal age-specific diagnostic test.

Beyond reasonable doubt it is known, that the use of cytology-based screening in the prophylaxis of cervical cancer is insufficient. Introduction of cervical cytology as the basis of cervical cancer screening during the second half of the twentieth century, contributed to a significant decline in the incidence and mortality rates due to cervical cancer world-wide. Nevertheless, despite the introduction of numerous modifications, sensitivity of this method in the diagnosis of high-grade squamous intraepithelial lesions (HSIL) is unsatisfying. Currently there is no question that there is a cause and effect relationship between persistent infection with high oncogenic types of HPV and the development of cervical intraepithelial neoplasia and its progression to invasive cancer. The development of knowledge of human papillomavirus and process of carcinogenesis lead to the incorporation of molecular tests, which can detect the DNA of high oncogenic types of HPV into diagnostic methods. Currently, there is an ongoing debate on the role of molecular diagnostic tests in the screening programs. Many authors underline that HPV testing is more sensitive for squamous intraepithelial lesions than cervical cytology. The American College of Obstetrics and Gynecology first incorporated HPV testing in their guidelines in 2003. Since 2012, HPV testing is highly recommended along with the routine cervical cytology for women age 30-65 years [5].

\section{OBJECTIVES}

The purpose of the study was to compare the diagnostic value of cervical cytology and HPV HR DNA testing for the diagnosis of low grade and high-grade squamous intraepithelial lesions across different age groups.

\section{MATERIAL AND METHODS}

Between 2011 and 2014, 1103 patients aged 25 and older underwent in-depth diagnostics at the Laboratory of Cervical Pathophysiology, Gynecology and Obstetrics Clinical Hospital, Karol Marcinkowski University of Medical Sciences, Poznan. Women were referred to the Laboratory because of either an abnormal Pap test result or a clinically suspicious cervical lesion. According to the Bethesda System (TBS), abnormal results include ASC-US, ASC-H, LSIL, HSIL, AGC or cancer cells. Incorrect cytologic results including LSIL and ASCUS were indication for directed punch biopsy in case of suspicious lesions presented in colposcopy examination. In case of AGC diagnosis, the colposcopy examination with punch biopsy of suspicious lesions was performed together with the curettage of endocervix and endometrium. The study excluded pregnant women and underage patients. The opinion of a Bioethical Committee has not been required to perform the study.

The number of patients with a particular cytological diagnosis is presented in Table 1.

The following tests were performed in each patient:

- Pap smear,

- HPV test,

- colposcopy examination,

- directed-biopsy of the cervix,

- histopathological evaluation.

\section{Pap smear}

The cytology was collected using a cyto-brush and smeared onto a microscope slide. Then, the cells were fixed and subsequently stained with haematoxylin, Orange G stain, as well as Eosin Azure. After staining and fixing the specimen, it was coated with xylene and Canada balsam. Smears were evaluated by senior cytologists and reported using currently recommended Bethesda System (TBS). All abnormal Pap test results were reevaluated by pathologists at the Laboratory of Pathophysiology of the Cervix, Gyne-

Table 1. Number of patients with a particular cytological diagnosis

\begin{tabular}{|l|c|c|c|c|c|c|c|}
\hline Pap test result & Normal & ASC-US & ASC-H & LSIL & HSIL & AGC & Suspicion of cancer \\
\hline Number of patients & 137 & 324 & 47 & 402 & 177 & 7 & 9 \\
\hline
\end{tabular}


cology and Obstetrics Clinical Hospital, Karol Marcinkowski University of Medical Sciences, Poznan.

\section{HPV test}

A material was collected from cervix using cyto-brush, and subsequently it was placed in a buffer, ThinPrep PreservCyt Solution. For detection of DNA HR HPV types, we used a molecular test known as the Cobas HPV test, Roche Diagnostics. This test, based on real-time PCR method, identifies 14 most commonly occurring high-risk, oncogenic, types of HPV in vitro with genotyping for high risk HPV types 16 and 18. Surveillance of the cells is guaranteed by simultaneous PCR amplification of the beta-globin HPV DNA.

\section{The colposcopy examination}

The colposcopy examination - conducted at the Laboratory of Cervical Pathophysiology - was performed using a stereoscopic colposcope, Olympus OCS-500. Visualization of the transformation zone was the basis for classification of the colposcopic image as satisfactory. Each time, 3\% acetic acid and Schiller's test using a Lugol's liquid was performed. Colposcopic images were evaluated using Reid's Colposcopic Index, which assesses lesion margin, color of acetowhitening, blood vessels and iodine staining.

\section{Directed-biopsy of the cervix}

In each patient, qualified for the study, a directed-biopsy of a clinically suspicious lesion and endocervical curettage were performed. Colposcopic image helped to identify abnormal cervical areas, which warranted biopsy. Sampled tissue, fixed in a buffer of formalin, after dehydration was embedded in paraffin blocks, and subsequently stained with hematoxylin and eosin. All specimens were subjected to a blinded independent evaluation of the Pathomorphological Laboratory team at Gynecology and Obstetrics Clinical Hospital, Karol Marcinkowski University of Medical Sciences, Poznan.

\section{Statistical methods}

In terms of diagnostic methods (cytodiagnostics and molecular test for the presence of DNA HR types HPV) we determined sample size with values of true positive, true negative, false positive and false negative. Based on these values, the sensitivity, specificity, positive and negative predictive value with $95 \%$ confidence intervals were estimated. The sensitivity and specificity of cytology and HPV were compared by McNemar's test while the PPV and NPV were compared by test for the two fractions. Four age groups were determined, in which these indicators were also calculated. It was estimated whether increasing age resulted in higher rates of sensitivity, specificity, PPV and NPV by Chi ${ }^{2}$ test for the trend. Statistical hypotheses were verified at the sig- nificance level $a=0.05$. Calculations were performed using the statistical package StatSoft, Inc. (2011) STATISTICA (data analysis software system), ver. 10 and MedCalc ver. 13,0,6,0.

\section{RESULTS}

The study included 1103 women. Pathomorphological results revealed the presence of high-grade squamous intraepithelial lesions - HSIL in 217 patients (19.6\%) and low-grade squamous intraepithelial lesions - LSIL in 169 patients (15.3\%). 13 cases of carcinoma planoepitheliale and 2 cases of adenocarcinoma were revealed which collectively constituted $1.3 \%$ of the studied population. 702 women (63.6\%) did not have a pathologically proven neoplastic lesions.

For the purpose of this study women were subdivided into four age groups:

- age 25-29 years - 306 women (27.7\%),

- age 30-39 years - 455 women (41.2\%),

- age 40-49 years - 194 women (17.5\%),

- 50+ age group - 148 women (13.4\%).

The population of individual subgroups is presented in Figure 1.

HSIL type of changes was most prevalent in all age groups. Over half of the patients aged 25-29 years and 30-39 years were diagnosed with HSIL type of changes (61.2\% and $53.4 \%$ respectively). In the remaining subgroups HSIL also constituted the most common diagnosis: $45.4 \%$ of patients aged $40-49$ years and $46.6 \%$ of patients over the age of 50 . All cases of adenocarcinoma and $84.6 \%$ of carcinoma planoepitheliale were diagnosed in women over the age of 40 years (Fig. 2)

Sensitivity of the Pap test and HPV test was calculated in each age group. The sensitivity of both tests for HSIL type of changes was the highest for women aged 40-49 years: cervical cytology $91.4 \%$ and HPV testing $97.1 \%$. For women under the age of 50, sensitivity of HPV testing was significantly higher than that of cervical cytology and clearly increased with the age of our patients. In the $50+$ age group, sensitivity

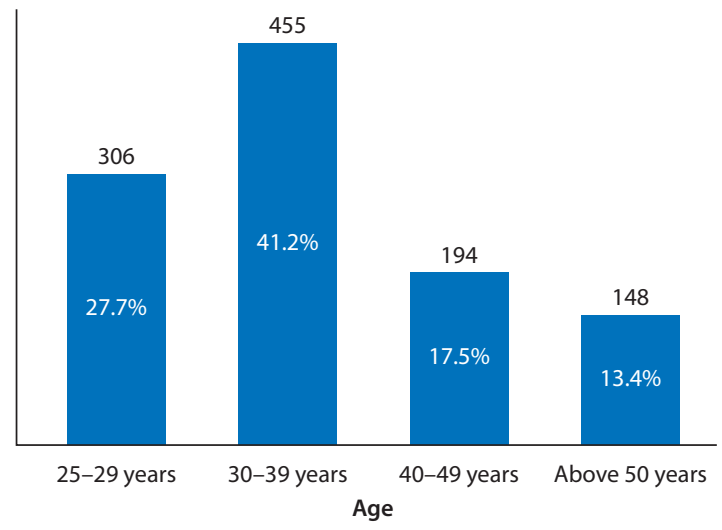

Figure 1. Population of women in each age group 


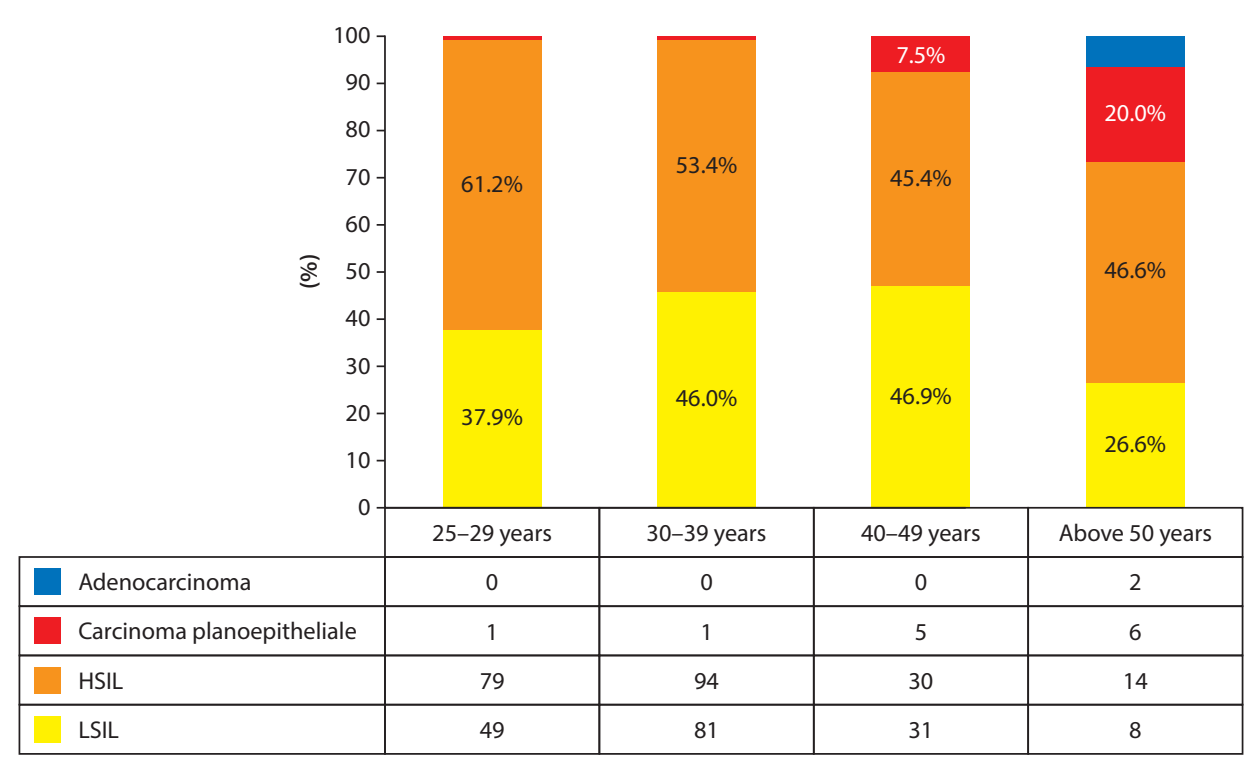

Figure 2. The incidence of histopathologic diagnoses in certain age groups

of the Pap testing was $4.6 \%$ higher than that of the HPV testing. The results are presented in both Table 2 and Figure 3.

Sensitivity values of both diagnostic tests for low-grade and high-grade squamous intraepithelial lesions collectively (LSIL + HSIL) again were highest in women aged $40-49$ years (90.9\% for HPV testing, 93.9\% for the Pap testing). The difference between sensitivity values of both tests faded in

\begin{tabular}{|c|c|c|c|c|}
\hline Age group & $25-29$ & $30-39$ & $40-49$ & $>50$ \\
\hline Group size & $N=306$ & $\mathrm{~N}=455$ & $N=194$ & $N=148$ \\
\hline Pap test & 87.5 & 86.3 & 91.4 & 90.9 \\
\hline HPV test & 93.7 & 95.7 & 97.1 & 86.3 \\
\hline
\end{tabular}

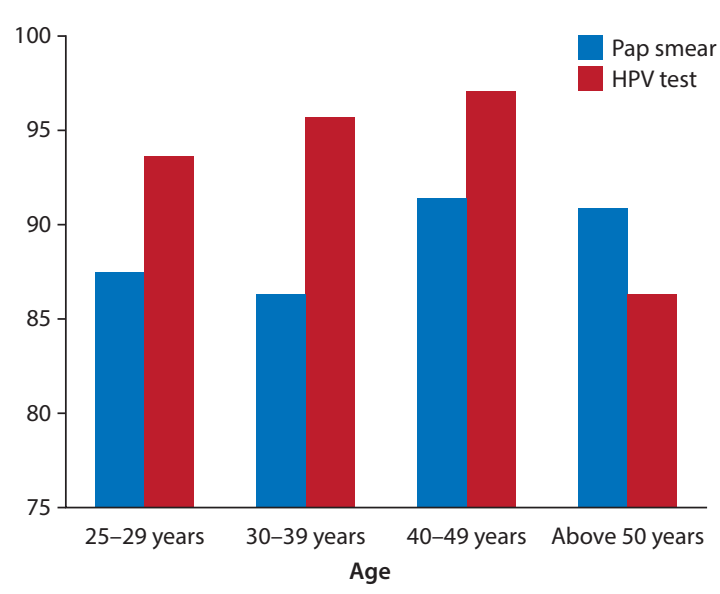

Figure 3. Sensitivity of Pap testing and HPV testing for HSIL across four age groups women under 50 years of age in favor of HPV testing, while in women aged 40-49 years sensitivity of high-risk HPV testing was $3 \%$ lower than that of the Pap testing. Meanwhile, in the $50+$ age group, as in the case of HSIL type of changes alone, sensitivity of cervical cytology was higher than that of HPV test. In the 50+ age group sensitivity of cervical cytology was $13.3 \%$ higher than that of molecular test (Tab. 3, Fig. 4).

Table 3. Sensitivity of Pap testing and HPV testing for LSIL + HSIL type of changes collectively across four age groups (\%)

\begin{tabular}{|l|c|c|c|c|}
\hline Age group & $25-29$ & $30-39$ & $40-49$ & $>50$ \\
\hline Group size & $\mathrm{N}=306$ & $\mathrm{~N}=455$ & $\mathrm{~N}=194$ & $\mathrm{~N}=148$ \\
\hline Pap test & 89.9 & 90.3 & 93.9 & 93.3 \\
\hline HPV test & 90.6 & 93.7 & 90.9 & 80.0 \\
\hline
\end{tabular}

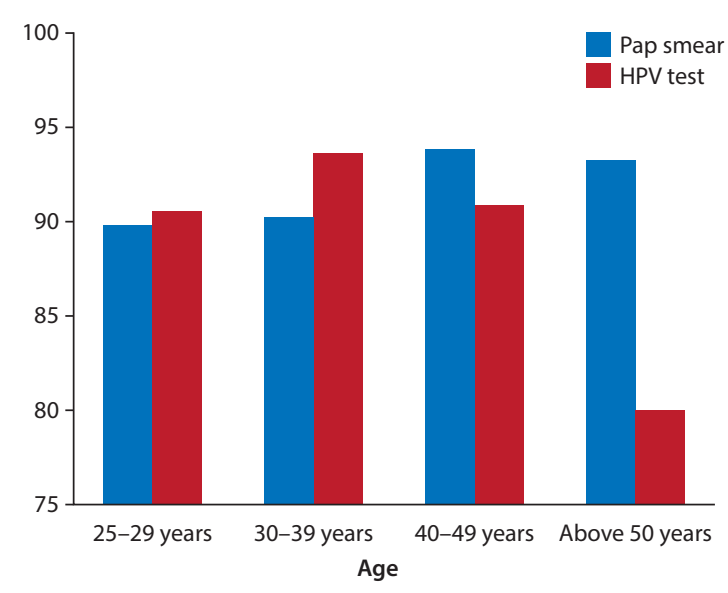

Figure 4. Sensitivity of Pap testing and HPV testing for LSIL + HSIL collectively across four age groups 


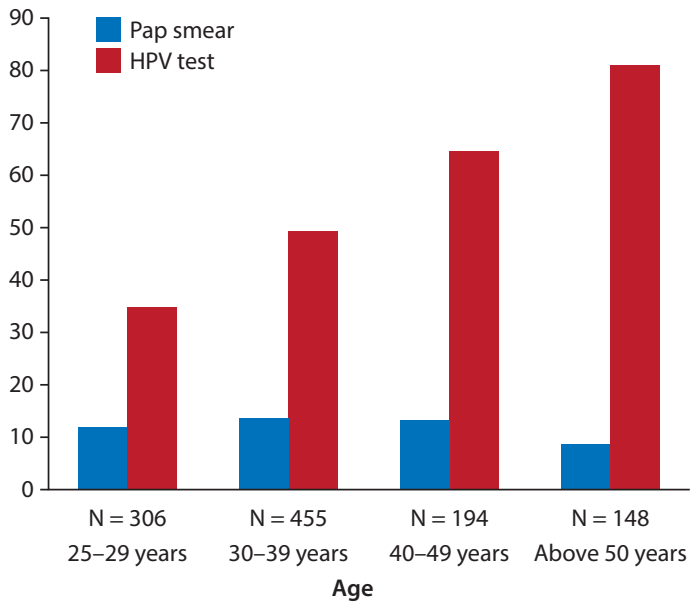

Figure 5. Specificity of Pap testing and HPV testing for HSIL across four age groups

Table 4. Specificity of Pap testing and HPV testing for HSIL across four age groups (\%)

\begin{tabular}{|l|c|c|c|c|}
\hline Age group & $25-29$ & $30-39$ & $40-49$ & $>50$ \\
\hline Group size & $\mathrm{N}=306$ & $\mathrm{~N}=455$ & $\mathrm{~N}=194$ & $\mathrm{~N}=148$ \\
\hline Pap test & 11.9 & 13.8 & 13.2 & 8.7 \\
\hline HPV test & 34.9 & 49.4 & 64.7 & 80.9 \\
\hline
\end{tabular}

Figures 5 and 6 show specificities of each method for HSIL and LSIL + HSIL, respectively. Specificity of a molecular test increases with the increasing age of women. Pap test specificity is, in both cases, smaller than that of HPV test. Furthermore, it is independent of patients' age (Tab. 4, 5).

\section{DISCUSSION}

The analysis of the age at diagnosis of cervical cancer in Poland, based on the Countries Cancer Register, revealed an increase in the number of observed cases at the age of 30 and older. The risk of cervical cancer increases with age, but mostly (about 60\%) concerns women age 45-64 years and then gradually decreases [6]. Intensification of preventive actions should focus on population of women aged 30-60 years. In the course of our study all cancer cases were diagnosed in women over the age of 40 . Along with increasing age the percentage of low-grade squamous intraepithelial lesions (LSIL) decreased, while the percentage of more advanced lesions like high-grade squamous intraepithelial lesions (LSIL) and cancers increased.

Numerous studies have shown that diagnostic test detecting the DNA of HR HPV types presents higher sensitivity and negative predictive value, compared to cervical cytology. A study conducted in Canada, analyzing ten thousand women, revealed that sensitivity of HPV testing for HSIL is $94.6 \%$, while sensitivity of cervical cytology is merely $55.4 \%$ [7]. Some reports show that sensitivity of Pap test-

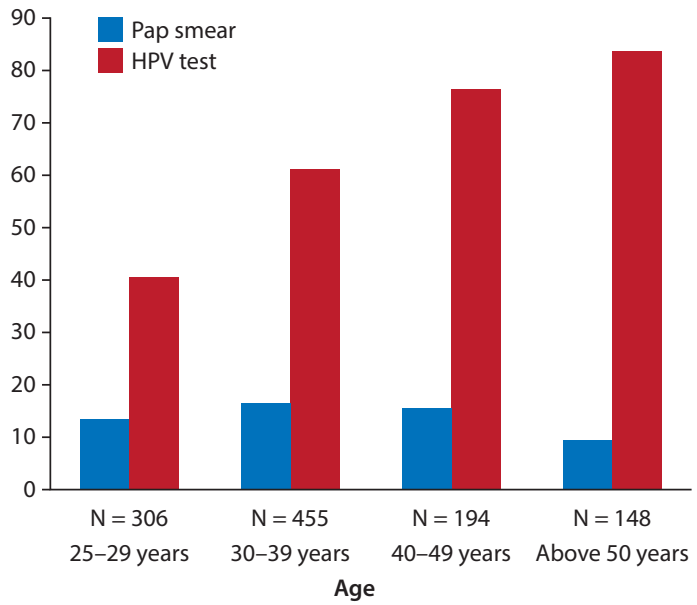

Figure 6. Specificity of Pap testing and HPV testing for LSIL + HSIL collectively across four age groups

Table 5. Specificity of Pap testing and HPV testing for LSIL + HSIL collectively across four age groups (\%)

\begin{tabular}{|l|c|c|c|c|}
\hline Age group & $25-29$ & $30-39$ & $40-49$ & $>50$ \\
\hline Group size & $\mathrm{N}=306$ & $\mathrm{~N}=455$ & $\mathrm{~N}=194$ & $\mathrm{~N}=148$ \\
\hline Pap test & 13.5 & 16.4 & 15.6 & 9.3 \\
\hline HPV test & 40.6 & 61.2 & 76.5 & 83.8 \\
\hline
\end{tabular}

ing increases with age [8]. According to Nanda et al., the range of sensitivity values of cervical cytology vary widely (30-87\%) [9]. Our study confirms that the molecular test is characterized by higher sensitivity, when compared to cervical cytology, for high-grade squamous intraepithelial lesions. Nevertheless, considering both low-grade and high-grade squamous intraepithelial lesions, sensitivity of both methods is comparable. Furthermore, it was noted that sensitivity value of HPV testing gradually increases with age and reaches a peak in women aged $40-49$ years. Whereas a relationship between increasing sensitivity of cervical cytology and age of our patients was not observed.

Diagnostic test with high specificity is useful for ruling out disease in healthy individuals. In the present study, in $63.6 \%$ of women who underwent in-depth diagnostic tests, mainly for epithelial cell abnormalities, the lesion of cervical epithelium have not been histologically confirmed. High rate of false positives can lead to unnecessary diagnostic and therapeutic interventions, and therefore increase the costs. Specificity for HSIL type of changes ranged between $8.7 \%$ and $13.8 \%$ and did not correlate with patients' age. Fundamentally different results were observed in the case of HPV test. Specificity of HPV test for HSIL as well as for HSIL plus LSIL collectively, gradually increased with age. In 50+ age group, specificity of molecular test was over $80 \%$, which seems to be the logical consequence of the natural course of HPV infection. Human papillomavirus infection is widely spread among young women. It is 
estimated that HPV infection concerns $24 \%$ of women aged 25 and younger while in women aged $35-44$ years it is only $9 \%$ [10]. Beyond that, in teenagers and women under 34 years of age, over $80 \%$ of precancerous lesions of the cervix regress spontaneously without treatment. In women aged 35 and above, $40 \%$ regress, while the remaining $60 \%$ progress to more advanced disease [11]. This data explain the low specificity of tests among younger age groups. Simultaneously, along with the increasing age, the risk of persistent infection increases, meanwhile the prevalence of the virus among the population decreases $[12,13]$. In medical and economic terms, it seems justifiable to introduce HPV tests into screening not earlier than in women aged 30-35.

\section{CONCLUSIONS}

1. Sensitivity values of cervical cytology and molecular test for high-grade squamous intraepithelial lesions were highest in women aged 40-49 years.

2. Sensitivity of HPV testing was higher than that of cervical cytology among women under age 50 .

3. Specificity of HPV test increased with age and was several-fold higher than specificity of cervical cytology across all age groups.

\section{REFERENCES}

1. Wojciechowska U, Didkowska J, Zachorowania i zgony na nowotwory złośliwe w Polsce. Krajowy Rejestr Nowotworów, Centrum Onkologii - Instytut im. Marii Skłodowskiej-Curie. http://onkologia.org.pl/raporty (28.02.2016).

2. Dyzman-Sroka A, Trojanowski M, Kubiak A, et al. Nowotwory złośliwe w Wielkopolsce w 2012 roku. In: Wielkopolski Rejestr Nowotworów. Biuletyn 11. Wielkopolskie Centrum Onkologii, Poznań. 2014
3. Herrington CS. The terminology of pre-invasive cervical lesions in the UK cervical screening programme. Cytopathology. 2015; 26(6): 346-350, doi: 10.1111/cyt.12307, indexed in Pubmed: 26767601.

4. Waxman A, Chelmow D, Darragh T, et al. Revised Terminology for Cervical Histopathology and Its Implications for Management of High-Grade Squamous Intraepithelial Lesions of the Cervix. Obstetrics \& Gynecology. 2012; 120(6): 1465-1471, doi: 10.1097/AOG.0b013e31827001d5.

5. Saslow D, Solomon D, Lawson HW, et al. American Cancer Society, American Society for Colposcopy and Cervical Pathology, American Society for Clinical Pathology, ACS-ASCCP-ASCP Cervical Cancer Guideline Committee. American Cancer Society, American Society for Colposcopy and Cervical Pathology, and American Society for Clinical Pathology screening guidelines for the prevention and early detection of cervical cancer. J Low Genit Tract Dis. 2012; 16(3): 175-204, doi: 10.1097/LGT.0b013e31824ca9d5, indexed in Pubmed: 22418039.

6. Wojciechowska U, Didkowska J. Nowotwory w Polsce w 2012 roku. Nowotwory. Journal of Oncology. 2013; 63(3): 197-216, doi: 10.5603/njo.2013.0001.

7. Mayrand $\mathrm{MH}$, et al. HPV testing vs Papanicolaou screening tests for cervical cancer. NE J Med. 2007; 357: 1579-1588.

8. Cuzick J, Clavel C, Petry KU, et al. Overview of the European and North American studies on HPV testing in primary cervical cancer screening. Int J Cancer. 2006; 119(5): 1095-1101, doi: 10.1002/ijc.21955, indexed in Pubmed: 16586444.

9. Nanda K, McCrory DC, Myers ER, et al. Accuracy of the Papanicolaou test in screening for and follow-up of cervical cytologic abnormalities: a systematic review. Ann Intern Med. 2000; 132(10): 810-819, indexed in Pubmed: 10819705.

10. Bruni L, Diaz M, Castellsagué $X$, et al. Cervical Human Papillomavirus Prevalence in 5 Continents: Meta-Analysis of 1 Million Women with Normal Cytological Findings. The Journal of Infectious Diseases. 2010; 202(12): 1789-1799, doi: 10.1086/657321.

11. van Oortmarssen GJ, Habbema JD. Epidemiological evidence for age-dependent regression of pre-invasive cervical cancer. Br J Cancer. 1991; 64(3): 559-565, indexed in Pubmed: 1911199.

12. Ho GY, Bierman R, Beardsley $L$, et al. Natural history of cervicovaginal papillomavirus infection in young women. N Engl J Med. 1998; 338(7): 423-428, doi: 10.1056/NEJM199802123380703, indexed in Pubmed: 9459645.

13. Beachler DC, Tota JE, Silver MI, et al. Trends in cervical cancer incidence in younger US women from 2000 to 2013. Gynecol Oncol. 2017; 144(2): 391-395, doi: 10.1016/j.ygyno.2016.11.031, indexed in Pubmed: 27894752. 